\title{
Luisa Puig
}

Seminario de Poética, IIFL, UNAM

\section{El discurso paradójico.}

Un análisis lingüístico argumentativo

...Por mucho que lo intentamos, no logramos aprehender la realidad de las cosas. Y puede que la terrible razón de eso esté en que lo único real sea su apariencia.

Oscar Wilde

(Entrevista en la cárcel de Reading)

Desde la antigüedad clásica, la retórica ha considerado que el lenguaje, y en particular el discurso, es un medio idóneo para elaborar razonamientos, hacer inferencias o presentar argumentos con el fin de convencer o persuadir al interlocutor de la justeza de una idea o de la necesidad de llevar a cabo una acción.

Refutar esta creencia parecería ilógico o impensable pero, desde un punto de vista lingüístico, es posible tener una concepcíón muy diferente de la lengua y de la manera como funciona. Así, se puede considerar que en los encadenamientos discursivos (que contienen conectores como por consiguiente, por lo tanto, entonces, sin embargo, etc.) no se realizan inferencias ni razonamientos. Tal es la posición que sostiene la semántica de los bloques argumentativos de Oswald Ducrot y Marion Carel. ${ }^{1}$ 2000.

${ }^{1}$ Para un análisis del desarrollo y de la evolución de esta teoría cf.: L. Puig, 
Esta teoría lingüístico argumentativa parte, pues, de una concepción del discurso opuesta a los planteamientos de la retórica clásica. Para esta última, las inferencias y los razonamientos ocupan un lugar primordial en la estrategia persuasiva, mientras que para la semántica de los bloques argumentativos, los razonamientos en el discurso son el resultado de una pura ilusión. ${ }^{2}$

Esta perspectiva implica, a la vez, una manera específica y particular de percibir y definir el sentido. Al respecto, cabe aclarar que en la semántica lingüística existen tantas concepciones del sentido como teorías que pretenden describirlo. Y para lograrlo, cada una de ellas toma el aspecto del sentido que le parece más pertinente: su valor referencial (en cuyo caso estaríamos hablando de una semántica denotativa), alguna propiedad del pensamiento que se expresa (una semántica cognitiva), una posibilidad inferencial (una semántica de tipo lógico), alguna función en la actividad de la comunicación (una semántica enunciativa) o los encadenamientos de las palabras y de las frases en el discurso (datos que son pertinentes para la semántica de los bloques argumentativos). ${ }^{3}$

Por consiguiente, es absurdo pensar que una descripción semántica pueda justificarse independientemente de la teoría que la explica, ya que los datos por describir son construcciones hipotéticas y, por lo tanto, la teoría determina lo que se va a observar. Esta situación (a la que está forzosamente condenado cualquier análisis que tenga que ver con el sentido) hace que el objetivo de una descripción semántica sólo pueda consistir en evaluar la relación que se da entre las decisiones que se toman a partir de una determinada teoría y la visión de los hechos que las diversas teorías implican. ${ }^{4}$

Una vez aclarada la relación que necesariamente guardan los datos observables y la teoría semántica, es oportuno especificar

2 O. Ducrot, 1998.
3 O. Ducrot, 1997.
${ }^{4}$ O. Ducrot, 1980: pp. 23 y s. 
que el propósito de la semántica de los bloques argumentativos de Ducrot y Carel es desterrar del discurso la pretendida descripción de la realidad, ${ }^{5}$ a fin de describir en términos no informativos, sino puramente argumentativos, los fenómenos de la lengua. En esta óptica, llevar a cabo una descripción semántica consistirá en estudiar el valor argumentativo de los enunciados, entendiendo por valor argumentativo sus posibles encadenamientos discursivos.

Esta concepción de la lengua y del análisis semántico se relaciona con otra proposición teórica igualmente importante: lo que hay tras las palabras son otras palabras, no un referente, ni la realidad, ni el mundo.

De igual manera, este punto de vista encierra una concepción polifónica del significado: en un enunciado hay una pluralidad de voces que se escuchan. El locutor pone en escena, en el interior de su propio discurso, un diálogo entre diversos enunciadores; construye, en su discurso, el habla del otro. ${ }^{6}$

Aun cuando estos planteamientos teóricos resulten para algunos muy innovadores y para otros simplemente insólitos - tanto por cuestionar que en el discurso se den razonamientos e inferencias, como por considerar que las palabras no se emplean de manera verídica sino atendiendo a la organización que imponen al discurso-, se basan en la ya muy antigua noción de valor de Saussure, es decir, en el principio según el cual la lengua es un sistema cuyos elementos, los signos, no tienen un carácter propio, fuera de las relaciones mutuas que establecen al interior del todo.

5 En otras palabras, esta teoría se opone a una semántica "veritativa" o "denotativa", la cual considera que el significado de una palabra equivale a las condiciones a las que un objeto debe satisfacer para poder ser designado por esa palabra y, a nivel de la frase, el significado corresponde a las relaciones que deben guardar los objetos que designan las palabras de esa frase para que ésta pueda considerarse verdadera. O. Ducrot, 1997.

${ }^{6}$ Ejemplos de análisis polifónicos se pueden encontrar en: O. Ducrot et al., 1980, O. Ducrot, 1995, L. Puig, 2000. 
La tesis saussuriana llevada a la semántica de los bloques argumentativos significa, pues, considerar, en primer lugar, que el sentido debe buscarse en el interior del orden lingüístico, en las relaciones que los signos guardan entre sí, sin tomarlo como un reflejo deformado o empobrecido de la realidad preexistente, ${ }^{7} \mathrm{y}$, en segundo lugar, afirmar que existe una interdependencia radical entre los diversos segmentos de un encadenamiento argumentativo. En otras palabras, el sentido de cada segmento contiene la indicación de que es argumento o conclusión de otro segmento.

Ahora bien, esta interdependencia no es la que puede darse, en un razonamiento, entre una conclusión y sus premisas. Tampoco se trata de una interdependencia semántica según la cual cada segmento del discurso forma parte de un mismo contexto.

Al hablar de una semántica de los bloques argumentativos sus autores consideran, como ya dije antes, que los encadenamientos argumentativos no tienen como función marcar una inferencia entre una afirmación y otra - aun cuando contengan conectores como por lo tanto o sin embargo-, porque los segmentos que aparecen como argumento y conclusión no son semánticamente independientes el uno del otro, ${ }^{8}$ sino que conforman un sentido cuando se toman juntos en la argumentación. Esto significa que los conectores califican una cosa o una situación por medio de un bloque semántico único: "el por lo tanto es un medio para describir y no para probar, justificar, volver verosímil."

Para ilustrar lo anterior veamos algunos ejemplos en los que resulta difícil aplicar un análisis de tipo inferencial:

7 O. Ducrot, 1997.

${ }^{8}$ En una inferencia, en cambio, se admite una proposición en virtud de su relación con otras proposiciones consideradas de antemano como verdaderas.

${ }^{9}$ O. Ducrot, 1998. 
1. Juan es un hombre extraordinario, por lo tanto le darán el Nobel de física.

2. Juan es un hombre extraordinario, por lo tanto rechazará el Nobel de física.

Aun cuando en ambos encadenamientos se relacionan dos enunciados asertivos por medio del conector por lo tanto, éstos no señalan una inferencia que vaya de una afirmación a la otra porque, para eso, sería necesario poder atribuir, a cada uno de los segmentos que une el conector, un sentido independiente del otro segmento.

En estos ejemplos es claro, en efecto, que cada segmento en 1. y en 2. no puede comprenderse sin tomar en cuenta que es argumento o conclusión del otro; cada afirmación contiene el conjunto del encadenamiento en el que se encuentra. El por lo tanto permite explicitar tanto la cualidad de Juan como el tipo de distinción o de reacción a la que se alude. Así, mientras que en 1 . su carácter extraordinario se relaciona con una capacidad intelectual sobresaliente, en 2., en cambio, la misma cualidad es de carácter moral. Por lo tanto, a pesar de que parece tratarse del mismo calificativo, es decir, del mismo argumento, las conclusiones en cada caso dan un sentido diferente a este calificativo: para ganar el Nobel de física es necesario ser científicamente muy distinguido, $y$, para rechazarlo, tener férreas convicciones personales.

De igual manera, las conclusiones se comprenden a partir de los dos sentidos de extraordinario en estos ejemplos. Así, la certeza al afirmar que le concederán a Juan el Nobel de física se explica por su inteligencia y sus conocimientos reconocidos, y la seguridad de que Juan lo rechazará también responde a que se le conoce por sus principios imperturbables. Tanto en 1. como en 2. se manifiesta, pues, un bloque semántico único: en el primer caso se trata de la capacidad intelectual que será premiada; en el segundo, del valor personal que Juan pondrá de 
manifiesto al rechazar el premio. Ninguno de estos discursos puede descomponerse semánticamente, porque los ejemplos en cuestión pretenden calificar una situación en su conjunto.

En otras palabras, los locutores de estos discursos no califican primero (y de igual manera) a una persona, para luego, a partir de una supuesta descripción objetiva, argumentar a favor de una determinada conclusión. El sentido mismo de los argumentos en 1. y en 2. está incompleto sin tal o cual conclusión. Pero la conclusión, a su vez, también necesita del argumento al que se articula para esclarecerse. ${ }^{10}$

Por esta razón, al enunciar

3. Juan no es un hombre extraordinario, por lo tanto le darán el Nobel de física

aun cuando las conclusiones de 1. y de 3. sean idénticas, no tienen el mismo valor semántico. La atribución del premio en 1. tiene que ver con el valor científico que se le reconoce a Juan y que lo hace digno de recibir uno de los galardones más prestigiados, mientras que en 3. esta atribución se relaciona con la falta de valor que el locutor atribuye a dicho premio, dado que se otorga a una persona que no es sobresaliente.

La decisión de considerar los encadenamientos conclusivos como la expresión de un bloque semántico que expresa un punto de vista único, concierne igualmente a discursos sin un conector explícito:

\section{Juan es extraordinario, le darán el Nobel de física.}

Por otra parte, también es posible constatar el mismo tipo de interdependencia entre las palabras de los dos segmentos (en este caso: entre ser extraordinario y obtener el Nobel o rechazarlo) en otro tipo de encadenamientos:

${ }^{10}$ M. Carel, 1994: p. 69; y M. Carel, O. Ducrot, 1999: p. 10. 
5. Si Juan es un hombre extraordinario, seguramente le darán el Nobel de física.

6. A Juan le darán el Nobel de física, ya que es un hombre extraordinario.

Estos ejemplos ilustran, pues, cómo la argumentación no se funda en inferencias sino que se encuentra enraizada en el léxico mismo. De hecho, los encadenamientos discursivos no hacen más que desarrollar las representaciones que ya contienen las palabras. En efecto, además de los encadenamientos que se realizan por medio de la gran diversidad de conectores que existen en las lenguas naturales (pero, además, incluso, etc.), las palabras (sustantivos, adjetivos, verbos) evocan, sugieren, en su sentido mismo, argumentaciones, ${ }^{11}$ ya que precisamente por medio de las palabras se categoriza y se piensa. ${ }^{12}$

Este tipo de descripción semántica concierne, entonces, a las llamadas palabras llenas (adjetivos, sustantivos, verbos) y, más allá de las palabras, a los enunciados, y concierne también al encadenamiento de las palabras y de los enunciados en el discurso. Por lo tanto, esta teoría no sólo es de carácter estructural sino también enunciativo, entendiendo por enunciativa la posibilidad de que las virtualidades argumentativas que poseen $y$ evocan las palabras y los enunciados, se sitúen en el discurso, es decir, como la continuación de un discurso anterior y como el anuncio de uno que le seguirá. ${ }^{13}$

Los encadenamientos conclusivos tienen su complemento en otro tipo de discursos: aquellos que contienen encadenamientos de tipo concesivo (ya sea que se expresen por medio

11 Así, en uno de los sentidos del calificativo extraordinario, por ejemplo, al decir, Juan es un hombre extraordinario, se condensan argumentaciones del tipo: "Esta acción es casi imposible de realizar, sin embargo Juan logrará llevarla a cabo."

12 M. Carel, 1994: pp. 66 y 80.

13 O. Ducrot, 1997. 
de locuciones adverbiales como sin embargo, o a pesar de, o de conjunciones como aun cuando o aunque).

Veamos los siguientes ejemplos:

7. Juan es un hombre extraordinario; sin embargo, no le darán el Nobel de fisica.

8. Juan es un hombre extraordinario; sin embargo, no rechazará el Nobel de fisica.

Aun cuando los discursos conclusivos o resultativos y los concesivos se oponen en cierta forma (es imposible asumir ambos a la vez), también poseen algo en común: tanto el locutor de 1. como el de 7. admiten que, por su capacidad intelectual y sus conocimientos científicos, Juan debería ganar el Nobel de física. Lo mismo sucede en 2. y 8.: ambos locutores consideran que, por sus principios inquebrantables, Juan rechazaría el galardón. Por consiguiente, los discursos en 7. y 8. expresan, respectivamente, los mismos bloques semánticos que se mànifiestan en los enunciados 1 . y $2 .{ }^{14} \mathrm{La}$ diferencia estribaría únicamente en el aspecto bajo el cual el locutor utiliza estos discursos: en 1. y 2. los emplea bajo su aspecto normativo, mientras que en 7. y en 8. bajo el transgresivo. ${ }^{15}$

Resumiendo, para esta teoría el rasgo que permite calificar un discurso como argumentativo no es una supuesta relación de justificación entre un argumento y su conclusión, sino la interdependencia que existe entre los segmentos de un encadenamiento. A su vez, estos encadenamientos pueden ser de dos tipos: los discursos normativos (cuyos encadenamientos se rea-

${ }^{14}$ La oposición entre bloques semánticos es patente, en cambio, al comparar, respectivamente, 1. y 2., con los dos siguientes ejemplos:

a. Juan es un hombre extraordinario; por lo tanto, no le darán el Nobel de fi sica.

b. Juan es un hombre extraordinario; por lo tanto, no rechazará el Nobel de fisica.

${ }^{15}$ M. Carel, 1995: p. 178. 
lizan por medio de conectores como por lo tanto, que abreviamos PT) y los transgresivos (cuyos encadenamientos comportan conectores del tipo de sin embargo, abreviado SE). Éstos son, para la semántica de los bloques argumentativos, los dos tipos fundamentales de argumentación.

Ahora bien, la asociación de una expresión y de un aspecto argumentativo (ya sea normativo o transgresivo) puede efectuarse a través de la lengua o en el discurso. En el primer caso se hablará de una argumentación intrínseca, $\mathrm{y}$, en el segundo, de una extrinseca. ${ }^{16}$

Así, por ejemplo, la lengua asocia intrínsecamente el adjetivo medroso, con los aspectos argumentativos peligro PT miedo, responsabilidad PT miedo, imprevisto PT miedo. ${ }^{17}$ Por definición, una persona medrosa es la que reacciona con temor ante cualquier situación de peligro, cualquier responsabilidad o, en general, ante cualquier acontecimiento imprevisto. Aquí se dirá que los aspectos argumentativos peligro PT miedo, responsabilidad PT miedo, imprevisto PT miedo son intrínsecos a la expresión que definen; pero, además, estos aspectos son internos $^{18}$ al sentido de la expresión en cuestión.

Por otra parte, la lengua también asocia el adjetivo medroso a discursos como 9. y 10.:

9. Jaime es un medroso; por lo tanto, no aceptaría la dirección de la compañía.

10. Jaime es un medroso; sin embargo, te defendería en un asalto.

${ }^{16}$ M. Carel, O. Ducrot, 1999: p. 15.

${ }^{17} \mathrm{El}$ adjetivo medroso podría asociarse a otros aspectos además de los ya mencionados. En efecto, el análisis a partir de los encadenamientos intrínsecos permite que se manifieste toda la capacidad argumentativa de las palabras y expresiones de la lengua.

${ }^{18} C f$. infra, la nota siguiente. 
En estos casos se dirá que los aspectos argumentativos medroso PT NO-aceptación de un cargo y medroso SE defensa son también intrínsecos, pero externos, ${ }^{19}$ de medroso.

En cambio, en otras ocasiones, la semántica de una palabra puede no estar en relación con el encadenamiento en el que se encuentra:

11. Jaime es un medroso; por lo tanto, buscará a Pedro mañana.

En este caso se hablará de la continuación extrínseca que puede tener una expresión en el discurso.

La semántica de los bloques argumentativos considera que la posibilidad de un encadenamiento extrínseco, bajo el aspecto normativo, permite, a la vez, que se dé el mismo encadenamiento bajo el aspecto transgresivo:

12. Jaime es un medroso; sin embargo, buscará a Pedro mañana.

En el caso de los encadenamientos intrínsecos, en cambio, la correspondencia entre el aspecto normativo y el transgresivo sólo es posible cuando a la transformación con SIN EMBARGO le sigue una negación (o, en el caso inverso, cuando se trata de un POR LO TANTO negativo, la transformación necesita de un SIN EMBARGO positivo). Así, el correspondiente de 9. sería 13:

13. Jaime es un medroso; sin embargo, aceptaría la dirección de la compañía.

19 Además de la distinción intrínseco / extrínseco, la semántica de los bloques argumentativos distingue dos maneras en las que una argumentación puede asociarse a una expresión. Cuando la expresión que se estudia figura en uno de los encadenamientos del aspecto argumentativo al que se asocia, se dirá que este aspecto pertenece a la argumentación externa de dicha expresión; en caso contrario, se dirá que dicho aspecto proviene de su argumentación interna. Ibidem: p. 13. 
Si, en cambio, no hay inversión de lo positivo en negativo, el encadenamiento opuesto a un encadenamiento intrínseco da como resultado un discurso paradójico. En este caso, 14. y 15. se oponen, respectivamente, a 9. y a 10 .:

14. Jaime es un medroso; sin embargo, no aceptaría la dirección de la compañía.

15. Jaime es un medroso; por lo tanto, te defendería en un asalto.

Llegados a este punto cabe preguntarse: ¿qué propósito se puede perseguir al elaborar una teoría semántico argumentativa? El físico francés Pierre Duhem afirmaba que los hechos de hoy se construyeron con las teorías de ayer. Para Ducrot esto es igualmente cierto en lo que concierne a los hechos lingüísticos. En efecto, la lengua actual se observa a través de las teorías de antaño y "aquel que construye una teoría espera que ésta permita un día construir nuevos hechos - es decir, ...introducir un nuevo sesgo en la observación, cambiar la percepción misma que se tiene del habla, llamar la atención sobre aspectos hasta entonces imperceptibles". Y al relacionar las teorías con los hechos que pretenden explicar, se modifica automáticamente la manera de vivir y de percibir la lengua, lo que demuestra que la actividad metalingüística no es exterior a la lengua misma. ${ }^{20}$

Una ilustración de lo anterior es la posibilidad que abre la semántica de los bloques argumentativos a una explicación de expresiones, enunciados y discursos cuyo sentido resulta, en cierta forma, opuesto a lo que podría esperarse (que más arriba llamamos paradójico) y que se puede encontrar $-\mathrm{y}$, de hecho, a menudo se encuentra- en el discurso.

En efecto, dado que un discurso puede continuar de cualquier manera, ya sea predecible o inimaginable, es necesario lograr explicar, dentro de la semántica de los bloques argumen-

${ }^{20}$ O. Ducrot, 1989: pp. 5-7. 
tativos, la existencia del discurso paradójico (y no sólo tildarlo de anormal anteponiéndole un asterisco), y mostrar que posee características lingüísticas propias que lo distinguen de los discursos no paradójicos. ${ }^{21}$

Estos casos se corresponden con uno de los problemas a los que cotidianamente se enfrenta cualquier descripción lingüística y, en particular, semántica: el de los contraejemplos, los cuales, cuando no es posible explicarlos en el interior de la teoría misma, restan validez a la descripción.

Concretamente, si no es posible explicar el funcionamiento de los encadenamientos paradójicos, tampoco se podría sustentar la noción de encadenamiento intrínseco, es decir, la idea de que ciertas expresiones de la lengua poseen una argumentación intrínseca.

Ahora bien, como es de esperarse, esta explicación debe coincidir con los lineamientos teóricos arriba expuestos, por lo que una definición retórica, y por lo tanto no discursiva (en el sentido que aquí se define), resultaría incongruente (ya que en esa perspectiva se hablaría de ideas o de creencias opuestas o contrarias a las comunes o a las socialmente aceptadas). Así, desde un punto de vista retórico, la paradoja es una "figura de pensamiento que altera la lógica de la expresión pues aproxima dos ideas opuestas y en apariencia irreconciliables, que manifestarían un absurdo si se tomaran al pie de la letra - razón por la que los franceses suelen describirla como 'opinión contraria a la opinión'- pero que contienen una profunda y sorprendente coherencia en su sentido figurado". 22

En una perspectiva discursiva, en cambio, para distinguir las expresiones paradójicas de las que no lo son, primero es necesario definir la noción de encadenamiento lingüísticamente

${ }^{21}$ Un estudio sistemático de las características lingüísticas que posee el discurso paradojico rebasaría los límites del presente artículo, por lo que nos limitaremos solamente a esbozarlas.

22 H. Beristáin, 1997: p. 387. 
"dóxico", ${ }^{23}$ y contraponerle la de encadenamiento lingüísticamente paradójico. Se considerará que un encadenamiento $\mathrm{E}$ es lingüísticamente dóxico (LD) si el aspecto al que pertenece (ya sea normativo o transgresivo) está ya inscrito en la significación intrínseca de uno de los segmentos de $\mathrm{E}^{24}$

Así, por ejemplo, 9. es LD porque, como ya vimos, el aspecto medroso PT NO-aceptación de un cargo está inscrito en la significación de Jaime es un medroso: alguien de naturaleza temerosa no toma a su cargo un puesto de responsabilidad. En cambio, 14. es lingüísticamente paradójico (LP) porque el aspecto medroso SE NO-aceptación de un cargo no es intrínseco a Jaime es un medroso.

Sistematizando lo anterior, es posible proponer un criterio para reconocer un encadenamiento lingüísticamente paradójico. Consideraremos " $a$ con $b$ " como un encadenamiento formado de un primer segmento $a$ y de un segundo segmento $b, \mathrm{y}$ " $a$ con' $b$ " como el encadenamiento resultado de la inversión del conector.

Para que un encadenamiento " $a$ con $b$ " sea lingüísticamente paradójico es necesario que " $a$ con $b$ " no sea LD y que " $a$ con' $b$ " sea LD. ${ }^{25}$

Veamos el discurso 15:

15. Jaime es un medroso; por lo tanto, te defendería en un asalto.

En primer lugar, 15. no es $\mathrm{LD}$ ya que medroso PT defensa no está inscrito en la significación de Jaime es un medroso. Por otra parte, el discurso 10:

${ }^{23}$ La elección de este apelativo responde a una necesidad de orden fonológico: poder contraponerlo a la noción de encadenamiento lingüísticamente paradójico, pero, además, este término alude a la "dóxa", es decir, al universo de la "opinión" de la retórica clásica y, por consiguiente, aquí resulta pertinente. Ducrot y Carel utilizan en francés los términos "enchaînement linguistiquement doxal". y "enchaînement linguistiquement paradoxal”.

${ }^{24}$ M. Carel, O. Ducrot, 1999: p. 16.

25 Idem: p. 17. 
resultado de la inversión del conector, es LD, por lo que 15. corresponde a un encadenamiento lingüísticamente paradójico.

Una vez definida la noción de encadenamiento paradójico, y con el fin de oponerse a la actitud que simplemente lo consideraría como "lingüísticamente inaceptable" o "incoherente", es necesario hacer hincapié en el sentido que puede tener un discurso paradójico.

Entre los rasgos que distinguen las palabras, ${ }^{26}$ enunciados o discursos paradójicos está su carácter polifónico, es decir, el hecho de que evocan implícitamente el discurso dóxico al cual se substituyen. Así, para contradecir que una persona medrosa no aceptaría un cargo de responsabilidad, el discurso16. alude implícitamente a este hecho:

16. Jaime es un medroso; por lo tanto, aceptaría la dirección de la compañía.

Esto en cambio no sucede en el caso de los discursos dóxicos, los cuales no evocan los discursos paradójicos subyacentes. ${ }^{27}$

La presencia muy frecuente de los encadenamientos paradójicos en el discurso es una muestra de la necesidad que tiene el hombre de cuestionar el sentido de las palabras y, con éste, las creencias o las instituciones sociales. ${ }^{28}$ Un ejemplo de esa necesidad sería la afirmación de Oscar Wilde, citada como epígrafe de este trabajo, en la que presenta una paradoja que, de hecho, no sólo aboga por las tesis que aquí se defienden, ${ }^{29}$ sino

${ }^{26}$ Como ejemplo de una palabra paradójica, Ducrot menciona el adjetivo en francés masochiste (en español, masoquista), el cual comporta, en su argumentación interna intrínseca, el aspecto sufrimiento Pr satisfacción. M. Carel, O. Ducrot, 1999: p. 23.

27 O. Ducrot, 1997.

${ }^{28}$ M. Carel, O. Ducrot, 1999: p. 19.

${ }^{29}$ En efecto, en este trabajo se sostiene que, aun cuando el discurso contenga razonamientos, éstos no tienen como función justificar nada y, por lo mismo, su carácter "inferencial" es ilusorio. 
que, además, ilustra esta idea contestataria, característica del discurso paradójico: "lo único real en la realidad de las cosas es su apariencia" (discurso al que, de acuerdo con la descripción anterior, correspondería el aspecto real por lo tanto aparente). ${ }^{30}$

Al mismo tiempo, los encadenamientos paradójicos también ilustran la manera como se puede contribuir al enriquecimiento semántico de la lengua. Así, cuando Wilde vuelve a afirmar:

...life is much too important a thing ever to talk seriously about $i t^{31}$

el aspecto muy importante por lo tanto NO-serio constituye un nuevo bloque argumentativo que se añade a los bloques en los que aparecen estas expresiones (por ejemplo, importancia / reflexión, importancia / interés, seriedad / virtud, seriedad / sensatez, etc.), lo que da lugar a nuevos discursos en los que las palabras "se transforman espectacularmente". Lo extraordinario de este procedimiento es que, para construir nuevos bloques argumentativos, se empieza por "echar abajo" los anteriores. ${ }^{32}$

En algunas ocasiones, el discurso paradójico puede dejar de serlo debido al contexto que le sigue. Por ejemplo, veamos el siguiente discurso en el que nuevamente habla Wilde:

Beauty has as many meanings as man has moods. Beauty is the symbol of symbols. Beauty reveals everything, because it

- ${ }^{30}$ Ahora bien, al hablar de la apariencia de algo, se puede uno referir tanto a su "aspecto exterior", como a "una cosa que parece y no es" (Diccionario de la Lengua Española). En inglés el sustantivo appearance también posee estos dos sentidos "the outward form somebody or something has" $y$ "what somebody or something appears to be though in fact they may not be" (Oxford Advanced Learner's Dictionary of Current English). Por consiguiente, el juego de ambos sentidos viene a ser la justificación ad hoc para la tesis de la semántica de los bloques argumentativos.

31 “...la vida es una cosa demasiado importante como para jamás hablar seriamente sobre ella". O. Wilde, 1969: p. 665.

${ }^{32}$ M. Carel, O. Ducrot, 1999: p. 20. 
expresses nothing. When it shows us itself, it shows us the whole fiery-coloured world. ${ }^{33}$

Aquí, el aspecto no expresar nada por lo tanto revelarlo $\underline{\text { todo }}^{34}$ corresponde a un discurso paradójico. Esta paradoja se aclara, sin embargo, enseguida, por lo que se trata sólo de una pseudoparadoja. Así, la relación original $a P T c$ (no expresar nada por lo tanto revelarlo todo) se modifica y, por el contexto, da lugar al análisis $b P T(a S E c)$ : al mostrarse ante nosotros, la belleza nos muestra el mundo entero en sus ardientes colores $(=b)$, por lo tanto [la belleza no expresa nada $(=a)$ y sin embargo lo revela todo $(=c)$ ]. ${ }^{35}$

Volviendo al objetivo que puede perseguir una teoría semántica, el hecho de considerar el discurso como la expresión de bloques argumentativos en los que se manifiesta la cristalización del sentido de las palabras, abre muchas perspectivas nuevas para el análisis semántico. Pero, además, permite tener una concepción diferente de la lengua: ésta ya no se revela como un instrumento de comunicación transparente y objetivo: su fuerza persuasiva no estriba en demostrar y justificar por medio de inferencias, sino que más bien es un medio que permite "engañar", "imponer", "dominar" con pseudodemostraciones que, como dice Barthes, ${ }^{36}$ aparecen siempre bajo dos formas: a través del autoritarismo de la aserción y del gregarismo de la repetición.

33 "La belleza tiene tantos sentidos como el hombre temperamentos. La belleza es el símbolo de los símbolos. La belleza revela todo porque no expresa nada. Cuando se nos muestra, nos muestra el mundo entero de ardientes colores". 0. Wilde, 1969: p. 1030.

${ }^{34}$ Es necesario recordar que, para la semántica de los bloques argumentativos, los encadenamientos por medio de conectores como ya que o porque, aun cuando evidentemente no son equivalentes a por lo tanto, establecen la misma interdependencia entre los grupos de palabras que encadenan, por lo que forman parte del aspecto aPT b. M.Carel, O. Ducrot, 1999: p. 11.

35 M. Carel, O. Ducrot, 1999: p. 25.

${ }^{36}$ R. Barthes, 1978: pp. 10 y s. 
De ahí la función del discurso paradójico, el cual, en tanto que procedimiento lingüístico que cuestiona y se rebela contra los bloques argumentativos existentes $\mathbf{y}$, a la vez, en su calidad de fuente de creación semántica de nuevos bloques argumentativos, es un medio para contrarrestar o paliar las maniobras veladas de la lengua.

\section{Bibliografia}

BARTHES, R., Leçon inaugurale, lección inaugural de la Cátedra de Semiología del Colegio de Francia, pronunciada el 7 de enero de 1977, París, Seuil, 1978.

Beristáin, H., Diccionario de Retórica y Poética, $8^{\mathrm{a}}$ ed., México, Ed. Porrúa, 1997.

CAREL, M., "L'argumentation dans le discours: argumenter n'est pas justifier", Langage et société, París, Maison des sciences de l'homme, No. 70, diciembre, 1994, pp. 61-81.

_- "Pourtant: argumentation by exception", Journal of Pragmatics, Amsterdam, North-Holland, Vol. 24, Nos. 1/2, julio, 1995, pp. 167-188.

_ - O. Ducrot, "Le problème du paradoxe dans une sémantique argumentative", Langue Française, París, Larousse, No. 123, 1999.

Crowther, J. (ed.), Oxford Advanced Learner's Dictionary of Current English, Inglaterra, Oxford University Press, 1998.

DuCROT, O., Logique, structure, énonciation. Lectures sur le langage, París, Minuit, 1989.

-, "Pour une description non-véritative du langage", Linguistics in the morning calm 3. Selected Papers from SICOL-1992, Seúl, Hanshin Publishing Company, 1995, pp 45-57.

_- "Une sémantique énonciative peut-elle être structurale?", $C o$ loquio de San Marín para el Centenario de la publicación del Ensayo de Semántica de Michel Bréal, 1997, en prensa.

_- "Sobre la argumentación como medio de persuasión", Primer Congreso Internacional de Retórica en México. El Horizonte Interdisciplinario de la Retórica, 1998, en prensa. 
—

PuIG, L., La realidad ausente. Teoría y análisis polifónicos de la argumentación, México, UNAM, 2000.

Real Academia Española, Diccionario de la lengua española, Madrid, Espasa Calpe, 1994.

WILDE, O., VERA or THE NIHILISTS. A drama in a Prologue, and Four Acts. Complete Works of Oscar Wilde, Introd. de Vyvyan Holland, Londres, Collins, 1969, pp. 647-688.

, The Critic as artist. Complete Works of Oscar Wilde, Introd. de Vyvyan Holland, Londres, Collins, 1969, pp. 1009-1059. 